(C) Revista de Matemática: Teoría y Aplicaciones 2020 27(2) : 367-382

CIMPA - UCR ISSN: 1409-2433 (PRINT), 2215-3373 (ONLINE)

DOI: https://doi.org/10.15517/rmta.v27i2.34430

\title{
FUNCIONES DE DULAC PARA MODELOS MATEMÁTICOS DE LA ECOLOGÍA
}

\section{DULAC'S FUNCTIONS FOR MATHEMATICAL ECOLOGICAL MODELS}

\author{
Emmanuel MendozA* $^{*}$ Osvaldo OsunA $^{\dagger}$ \\ GEISER VILLAVICENCIO-PULIDO ${ }^{\ddagger}$
}

Received: 6/Nov/2018; Revised: 16/Jul/2019;

Accepted: 6/Feb/2020

Revista de Matemática: Teoría y Aplicaciones is licensed under a Creative Commons Reconocimiento-NoComercial-Compartirigual 4.0 International License.

Creado a partir de la obra en http://www.revistas.ucr.ac.cr/index.php/matematica

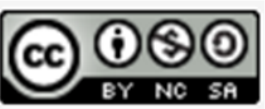

*Universidad Autónoma Metropolitana Unidad Iztapalapa, División de Ciencias Básicas e Ingeniería, Departamento de Matemáticas Aplicadas e Industriales, Ciudad de México, México. E-Mail: mennym962@gmail.com

${ }^{\dagger}$ Universidad Michoacana, Instituto de Física y Matemáticas, Morelia, Michoacán, México. E-Mail: osvaldo@ifm.umich.mx

${ }^{\ddagger}$ Universidad Autónoma Metropolitana Unidad Lerma, División de Ciencias Biológicas y de la Salud, Departamento de Ciencias Ambientales, Lerma de Villada, Estado de México, México. E-Mail: j.villavicencio@correo.ler.uam.mx 


\begin{abstract}
Resumen
Excluir la existencia de oscilaciones sostenidas en modelos de la ecología matemática es de vital interés para conocer la dinámica poblacional de especies interactuando. En este trabajo se construyen funciones de Dulac para algunas generalizaciones de modelos usados comúnmente en la ecología matemática. Dicho resultado excluye la existencia de órbitas periódicas para algunos modelos que describen competencia intraespecífica o interespecífica, relaciones de competencia, de depredación y captura de individuos de alguna de las especies.
\end{abstract}

Palabras clave: Funciones de Dulac; órbitas periódicas; modelos de competencia; modelos con mutualismo; modelos con captura.

\begin{abstract}
To exclude the existence of sustained oscillations in mathematical ecology models is of vital interest to know the populational dynamics of interacting species. In this paper, Dulac functions are constructed for some generalizations of models commonly used in populational ecology. This result excludes the existence of periodic orbits for some models that describe intraspecific and / or interspecific competition, relationships of competition, predation and capture of individuals of any of the species.
\end{abstract}

Keywords: Dulac's functions; periodic orbits; models with competence; models with mutualism; models with capture.

Mathematics Subject Classification: 34A34, 34C25.

\title{
1 Introducción
}

La construcción de funciones de Dulac es un problema que permanece abierto. Esto se debe a que la construcción de una función de Dulac depende totalmente del campo vectorial asociado al sistema de ecuaciones diferenciales ordinarias al cual se le quiere construir dicha función. En este sentido en el año 1988, E. Sáenz y I. Szánto mostraron un criterio para la construcción de cierto tipo de funciones de Dulac, para lo cual usaron métodos utilizados para la construcción de funciones de Lyapunov (ver [9]). Con este antecedente es que en tiempos recientes el interés por la construcción de funciones de Dulac ha resurgido. Así en el año 2011, Cherkas y Grin presentaron un método para la construcción de funciones de Dulac para sistemas de ecuaciones diferenciales polinomiales, mientras que Osuna y Villaseñor en el mismo año presentaron un método alternativo para la construcción de funciones de Dulac (ver [1,6]). En el método 
propuesto por Osuna y Villaseñor se cambia el problema de encontrar una función de Dulac para un sistema de ecuaciones diferenciales por el problema de encontrar una solución a una ecuación diferencial parcial, cuya solución es una función de Dulac para el sistema analizado. Algunas generalizaciones de esos resultados pueden encontrarse en [6], [7] y [8].

Por otra parte, construir funciones de Dulac para modelos de la biomatemática es relevante desde dos puntos de vista. En primer lugar, su existencia excluye la existencia de ciclos límites, los cuales pueden ser asociados a escenarios que pueden ser benéficos o perjudiciales para las poblaciones. Por ejemplo, la periodicidad del ciclo límite puede ser una estrategia que favorezca la reproducción de una especie (ver $[2,3,12]$ ) o la periodicidad del ciclo límite puede ser un fenómeno no deseable cuando el ciclo límite tiene amplitud muy grande y un periodo muy largo ya que durante un tiempo suficientemente grande el número de individuos en la población está en niveles muy bajos, lo que puede llevar a la población a la extinción, siendo este escenario catastrófico para la población. En segundo lugar, la construcción de una función de Dulac para un sistema de ecuaciones diferenciales puede utilizarse junto con el teorema de Poincaré-Bendixon para demostrar estabilidad global asintótica de las soluciones del sistema. En el caso que nos ocupa, en modelos de la biomatemática, demostrar estabilidad de los estados de equilibrio es fundamental para el diseño de estrategias que impacten en los escenarios poblacionales.

En la construcción de modelos de la ecología matemática se tienen presentes algunas características de poblaciones que comparten espacio y tiempo. Por ejemplo, si individuos de la misma especie o individuos de especies distintas compiten por los recursos. Particularmente, la competencia intraespecífica es la que se da cuando individuos de la misma especie compiten por los recursos, mientras que si individuos de diferentes especies compiten por los recursos esa competencia es llamada competencia interespecífica. Además de estas características existen relaciones entre las especies, por ejemplo relaciones del tipo presa-depredador. Otro tipo de relación entre especies es una relación de mutualismo, en la cual ambas especies se benefician y mejoran su aptitud biológica al interactuar entre ellas.

Lo expuesto anteriormente es lo que le da dirección a la investigación presentada en este trabajo cuyos resultados buscan aportar conocimiento sobre la dinámica de algunos modelos de la ecología de poblaciones. Para esto, en la sección 2 se muestran modelos utilizados en la ecología matemática, donde se tienen dos especies con competencia intraespecífica o interespecífica, así como relaciones del tipo presa-depredador, competencia o relaciones de mutualismo, además de términos que describen un proceso de captura de individuos de alguna 
de las especies. Finalmente, se construyen funciones de Dulac para algunas de sus posibles generalizaciones. En la sección 3 se muestra la aplicación de los resultados en modelos utilizados en la ecología matemática. Por último, en la sección 4 se discuten los resultados del trabajo.

\section{Algunos modelos clásicos de interacciones entre especies}

En esta sección se presentan algunos modelos de la biomatemática, los cuales consisten de sistemas de ecuaciones diferenciales ordinarias autónomas en dos dimensiones. En los ejemplos propuestos en esta sección se consideran dos especies, las cuales comparten tanto espacio como recursos, por lo que pueden existir interacciones entre sus individuos tales como competencia intraespecífica o interespecífica, depredación, mutualismo. Además, se considera un proceso de captura de individuos de una especie. Finalmente, se construirán funciones de Dulac para cada una de las generalizaciones de los modelos presentados y así descartar la existencia de órbitas periódicas.

Los modelos a considerar se presentan a continuación,

a) Considérese el modelo fitoplancton-zooplancton analizado en [10]:

$$
\begin{aligned}
& \dot{p}=r p\left[1-\frac{p}{k}\right]-\alpha z p, \\
& \dot{z}=\beta p z-c z-\frac{\theta p}{\gamma+p} z .
\end{aligned}
$$

donde $p(t)$ es la densidad de fitoplancton y $z(t)$ la densidad de zooplancton, $r$ es la tasa de crecimiento intrínseco del fitoplancton, $k$ es la capacidad de carga del fitoplancton, $\alpha$ es la tasa de depredación específica del zooplancton sobre el fitoplancton, $\beta$ representa el radio de biomasa consumida por el zooplancton para la producción de nuevo zooplancton, $c$ es la tasa de muerte de zooplancton, $\theta$ es la tasa de reproducción de toxina por especie de fitoplancton y $\gamma$ es la constante de saturación media del zooplancton.

b) Considérese el siguiente modelo de competencia y captura. En el siguiente modelo se puede suponer que ambas especies se depredan una a la otra, o que las dos especies sobreviven en el mismo hábitat con los mismos recursos. Este modelo puede describir ovejas y vacas pastoreando en el mismo sitio (ver [13]),

$$
\begin{aligned}
& \dot{x_{1}}=x_{1}\left(r_{1}-k_{1} x_{1}\right)-b_{1} x_{1} x_{2}-h_{1} x_{1}, \\
& \dot{x_{2}}=x_{2}\left(r_{2}-k_{2} x_{2}\right)-b_{2} x_{1} x_{2}-h_{2} x_{2} .
\end{aligned}
$$


En este caso el subíndice $i$ describe a la especie $i$, donde $i=1,2 . \quad r_{i}$ es la tasa de crecimiento intrínseco para la especie $i . k_{i}$ es la tasa de competencia intraespecífica. $b_{i}$ describe la competencia entre individuos de ambas especies. Finalmente, la tasa de cosecha de la especie $i$ está dada por $h_{i}$.

c) El siguiente ejemplo es un modelo básico de mutualismo facultativo [4]. En el cual las tasas de crecimiento intrínseco de la especie $i$ está denotada por $r_{i} . b_{i}$ es la tasa a la que se beneficia la especie $i$ de la presencia de la especie $j$, donde $i \neq j$. El parámetro $k_{i}$ describe el retardo en el tiempo para el cual el beneficio de las interacciones entre individuos de especies distintas afecta positivamente a la especie $i$,

$$
\begin{aligned}
& \dot{x_{1}}=r_{1} x_{1}\left[1-\frac{x_{1}}{k_{1}+b_{1} x_{2}}\right], \\
& \dot{x_{2}}=r_{2} x_{2}\left[1-\frac{x_{2}}{k_{2}+b_{2} x_{1}}\right] .
\end{aligned}
$$

Para construir una generalización de los sistemas (1), (2) y (3) considérense polinomios $p_{l}\left(x_{i}\right)$ para $l=1, \ldots, 8$ en las variables $x_{1}$ y $x_{2}$, los cuales están dados por:

$$
\begin{aligned}
& p_{1}\left(x_{1}\right)=a_{1} x_{1}+a_{2} x_{1}^{2}+a_{3} x_{1}^{3}+\cdots+a_{n} x_{1}^{n}, \\
& p_{2}\left(x_{1}\right)=b_{1} x_{1}+b_{2} x_{1}^{2}+b_{3} x_{1}^{3}+\cdots+b_{s} x_{1}^{s}, \\
& p_{3}\left(x_{1}\right)=c_{1} x_{1}+c_{2} x_{1}^{2}+c_{3} x_{1}^{3}+\cdots+c_{t} x_{1}^{t}, \\
& p_{4}\left(x_{1}\right)=d_{1} x_{1}+d_{2} x_{1}^{2}+d_{3} x_{1}^{3}+\cdots+d_{z} x_{1}^{z}, \\
& p_{5}\left(x_{1}\right)=e_{1} x_{1}+e_{2} x_{1}^{2}+e_{3} x_{1}^{3}+\cdots+e_{m} x_{1}^{m}, \\
& p_{6}\left(x_{2}\right)=h_{1} x_{2}+h_{2} x_{2}^{2}+h_{3} x_{2}^{3}+\cdots+h_{w} x_{2}^{w}, \\
& p_{7}\left(x_{2}\right)=g_{1} x_{2}+g_{2} x_{2}^{2}+g_{3} x_{2}^{3}+\cdots+g_{q} x_{2}^{q}, \\
& p_{8}\left(x_{2}\right)=k_{1} x_{2}+k_{2} x_{2}^{2}+k_{3} x_{2}^{3}+\cdots+k_{r} x_{2}^{r},
\end{aligned}
$$

donde los coeficientes son no negativos para todos los polinomios.

A continuación se probará que cada generalización de los modelos presentados en a), b) y c) admite una función de Dulac.

Teorema 1 Los sistemas:

a')

$$
\begin{aligned}
& \dot{x_{1}}=r x_{1}\left[1-\frac{p_{1}\left(x_{1}\right)}{k}\right]-\alpha x_{2} p_{2}\left(x_{1}\right), \\
& \dot{x_{2}}=\beta p_{3}\left(x_{1}\right) x_{2}-c p_{6}\left(x_{2}\right)-\frac{\theta p_{4}\left(x_{1}\right)}{\gamma+p_{5}\left(x_{1}\right)} p_{7}\left(x_{2}\right) .
\end{aligned}
$$


b')

$$
\begin{aligned}
& \dot{x_{1}}=x_{1}\left(r_{1}-k_{1} p_{1}\left(x_{1}\right)-b_{12} p_{6}\left(x_{2}\right)\right)-h_{1} p_{2}\left(x_{1}\right), \\
& \dot{x_{2}}=x_{2}\left(r_{2}-k_{2} p_{7}\left(x_{2}\right)-b_{21} p_{3}\left(x_{1}\right)\right)-h_{2} p_{8}\left(x_{2}\right) .
\end{aligned}
$$

c')

$$
\begin{aligned}
& \dot{x_{1}}=x_{1} r_{1}\left[1-\frac{p_{1}\left(x_{1}\right)}{k_{1}+b_{1} p_{6}\left(x_{2}\right)}\right]-b p_{2}\left(x_{1}\right), \\
& \dot{x_{2}}=x_{2} r_{2}\left[1-\frac{p_{7}\left(x_{2}\right)}{k_{2}+b_{2} p_{3}\left(x_{1}\right)}\right]-c p_{8}\left(x_{2}\right) .
\end{aligned}
$$

admiten una función de Dulac para todo $x_{1} \geq 0$ y $x_{2} \geq 0$ y por lo tanto el sistema no tiene órbitas periódicas.

Demostración. Para construir una función de Dulac para el modelo $\left.a^{\prime}\right)$ se utilizará el resultado del teorema 2 mostrado en el apéndice.

Sea

$$
\begin{aligned}
& \dot{x_{1}}=r x_{1}\left[1-\frac{p_{1}\left(x_{1}\right)}{k}\right]-\alpha x_{2} p_{2}\left(x_{1}\right), \\
& \dot{x_{2}}=\beta p_{3}\left(x_{1}\right) x_{2}-c p_{6}\left(x_{2}\right)-\frac{\theta p_{4}\left(x_{1}\right)}{\gamma+p_{5}\left(x_{1}\right)} p_{7}\left(x_{2}\right) .
\end{aligned}
$$

Defínase

$$
f_{1}=r x_{1}\left[1-\frac{p_{1}\left(x_{1}\right)}{k}\right]-\alpha x_{2} p_{2}\left(x_{1}\right)
$$

$\mathrm{y}$

$$
f_{2}=\beta p_{3}\left(x_{1}\right) x_{2}-c p_{6}\left(x_{2}\right)-\frac{\theta p_{4}\left(x_{1}\right)}{\gamma+p_{5}\left(x_{1}\right)} p_{7}\left(x_{2}\right)
$$

Cálculando el negativo de la divergencia del campo vectorial asociado al sistema $\left.a^{\prime}\right)$ se tiene

$$
\begin{aligned}
-\operatorname{div}\left(f_{1}, f_{2}\right)= & -r\left[1-\frac{a_{1}+a_{2} x_{1}+a_{3} x_{1}^{2} \cdots+a_{n} x_{1}^{n-1}}{k}\right]+ \\
& +\frac{r}{k} \sum_{j=2}^{n}(j-1) a_{j} x_{1}^{j-1}+\alpha x_{2}\left[\frac{p_{2}\left(x_{1}\right)}{x_{1}}+\sum_{j=2}^{s}(j-1) b_{j} x_{1}^{j-1}\right]+ \\
& \left.-\beta p_{3}\left(x_{1}\right)+c\left[\frac{p_{6}\left(x_{2}\right)}{x_{2}}\right]+\sum_{k=2}^{w}(k-1) h_{k} x_{2}^{k-1}\right]+ \\
& +\frac{\theta p_{4}\left(x_{1}\right)}{\gamma+p_{5}\left(x_{1}\right)}\left[\frac{p_{7}\left(x_{2}\right)}{x_{2}}+\sum_{k=2}^{q}(k-1) g_{k} x_{2}^{k-1}\right] .
\end{aligned}
$$


Después de algunas factorizaciones se tiene que

$$
\begin{aligned}
-\operatorname{div}\left(f_{1}, f_{2}\right)= & -\frac{1}{x_{1}} f_{1}-\frac{1}{x_{2}} f_{2}+\frac{r}{k} \sum_{j=2}^{n}(j-1) a_{j} x_{1}^{j-1}+c \sum_{k=2}^{w}(k-1) h_{k} x_{2}^{k-1}+ \\
& +\alpha x_{2} \sum_{j=2}^{s}(j-1) b_{j} x_{1}^{j-1}+\frac{\theta p_{4}\left(x_{1}\right)}{\gamma+p_{5}\left(x_{1}\right)} \sum_{k=2}^{q}(k-1) g_{k} x_{2}^{k-1} .
\end{aligned}
$$

La función $c\left(x_{1}, x_{2}\right)$ se define como

$$
\begin{aligned}
c\left(x_{1}, x_{2}\right)= & -\frac{r}{k} \sum_{j=2}^{n}(j-1) a_{j} x_{1}^{j-1}-\frac{\theta p_{4}\left(x_{1}\right)}{\gamma+p_{5}\left(x_{1}\right)} \sum_{k=2}^{q}(k-1) g_{k} x_{2}^{k-1}+ \\
& -\alpha x_{2} \sum_{j=2}^{s}(j-1) a_{j} x_{1}^{j-1}-c \sum_{k=2}^{w}(k-1) h_{k} x_{2}^{k-1} .
\end{aligned}
$$

Utilizando $c\left(x_{1}, x_{2}\right)$ se construye la siguiente ecuación diferencial parcial

$$
f_{1} \frac{\partial \Phi}{\partial x_{1}}+f_{2} \frac{\partial \Phi}{\partial x_{2}}=\Phi\left(c\left(x_{1}, x_{2}\right)-\operatorname{div}\left(f_{1}, f_{2}\right)\right),
$$

cuya solución es una función de Dulac.

Sea $\Phi=h(z)$, donde $z=z\left(x_{1}, x_{2}\right)$, entonces se tiene que

$$
\left[f_{1} \frac{\partial z}{\partial x_{1}}+f_{2} \frac{\partial z}{\partial x_{2}}\right] \frac{\partial h}{\partial z}=h(c-d i v F) .
$$

Así que

$$
\begin{gathered}
\frac{\frac{\partial h}{\partial z}}{h}=\frac{(c-\operatorname{div} F)}{\left[f_{1} \frac{\partial z}{\partial x_{1}}+f_{2} \frac{\partial z}{\partial x_{2}}\right]}, \\
\frac{\partial \ln h}{\partial z}=\frac{-\frac{x_{2} f_{1}+x_{1} f_{2}}{x_{1} x_{2}}}{\left[f_{1} x_{2}+f_{2} x_{1}\right]}, \\
\frac{\partial \ln h}{\partial z}=-\frac{1}{z} .
\end{gathered}
$$

Por lo tanto,

$$
h\left(x_{1}, x_{2}\right)=\frac{1}{x_{1} x_{2}},
$$

es una función de Dulac para el sistema (8). 
Para demostrar el caso $b^{\prime}$ ) se procederá de manera análoga al caso demostrado anteriormente. En este caso, se construirá una función de Dulac para el sistema polinomial dado por

$$
\begin{aligned}
& \dot{x_{1}}=x_{1}\left(r_{1}-k_{1} p_{1}\left(x_{1}\right)-b_{12} p_{6}\left(x_{2}\right)\right)-h_{1} p_{2}\left(x_{1}\right), \\
& \dot{x_{2}}=x_{2}\left(r_{2}-k_{2} p_{7}\left(x_{2}\right)-b_{21} p_{3}\left(x_{1}\right)\right)-h_{2} p_{8}\left(x_{2}\right) .
\end{aligned}
$$

Defínase

$$
f_{1}=x_{1}\left(r_{1}-k_{1} p_{1}\left(x_{1}\right)-b_{12} p_{6}\left(x_{2}\right)\right)-h_{1} p_{2}\left(x_{1}\right)
$$

$\mathrm{y}$

$$
f_{2}=x_{2}\left(r_{2}-k_{2} p_{7}\left(x_{2}\right)-b_{21} p_{3}\left(x_{1}\right)\right)-h_{2} p_{8}\left(x_{2}\right) .
$$

El negativo de la divergencia del sistema está dada por:

$$
\begin{aligned}
-\operatorname{div}\left(f_{1}, f_{2}\right)= & -r_{1}+k_{1} p_{1}\left(x_{1}\right)+b_{12} p_{6}\left(x_{2}\right)+x_{1} p_{1}^{\prime}\left(x_{1}\right) k_{1}+p_{2}^{\prime}\left(x_{1}\right) h_{1} \\
& -r_{2}+k_{2} p_{7}\left(x_{2}\right)+b_{21} p_{3}\left(x_{1}\right)+x_{2} k_{2} p_{7}^{\prime}\left(x_{2}\right)+h_{2} p_{8}^{\prime}\left(x_{2}\right) .
\end{aligned}
$$

Nótese que:

$$
\begin{aligned}
& p_{1}^{\prime}\left(x_{1}\right)=\left(a_{1}+a_{2} x_{1}+a_{3} x_{1}^{2}+\cdots+a_{n} x_{1}^{n-1}\right)+\sum_{i=2}^{n}(i-1) a_{i} x_{1}^{i-1}, \\
& p_{2}^{\prime}\left(x_{1}\right)=\left(b_{1}+a_{2} x_{1}+b_{3} x_{1}^{2}+\cdots+b_{s} x_{1}^{s-1}\right)+\sum_{k=2}^{s}(k-1) b_{k} x_{1}^{k-1}, \\
& p_{7}^{\prime}\left(x_{2}\right)=\left(g_{1}+g_{2} x_{1}+g_{3} x_{1}^{2}+\cdots+g_{s} x_{2}^{s-1}\right)+\sum_{k=2}^{q}(k-1) g_{k} x_{2}^{k-1}, \\
& p_{8}^{\prime}\left(x_{2}\right)=\left(k_{1}+k_{2} x_{1}+k_{3} x_{1}^{2}+\cdots+k_{t} x_{2}^{t-1}\right)+\sum_{j=2}^{z}(j-1) b_{j} k_{2}^{j-1} .
\end{aligned}
$$

Para reducir las expresiones algebraicas se proponen los siguientes cambios de variables

$$
\begin{aligned}
a_{1}+a_{2} x_{1}+a_{3} x_{1}^{2}+\cdots+a_{n} x_{1}^{n-1} & =t^{n-1}\left(x_{1}\right), \\
b_{1}+a_{2} x_{1}+b_{3} x_{1}^{2}+\cdots+b_{s} x_{1}^{s-1} & =z^{s-1}\left(x_{1}\right), \\
g_{1}+g_{2} x_{1}+g_{3} x_{1}^{2}+\cdots+g_{s} x_{2}^{s-1} & =w^{s-1}\left(x_{2}\right), \\
k_{1}+k_{2} x_{1}+k_{3} x_{1}^{2}+\cdots+k_{t} x_{2}^{t-1} & =m^{t-1}\left(x_{2}\right) .
\end{aligned}
$$


Así que la expresión (11) puede escribirse como

$$
\begin{aligned}
-\operatorname{div}\left(f_{1}, f_{2}\right)= & -\frac{1}{x_{1}}\left[x_{1}\left(r_{1}-k_{1} t^{n-1}\left(x_{1}\right)-b_{12} p\left(x_{2}\right)\right)-h_{1} x_{1} z^{s-1}\left(x_{1}\right)\right] \\
& -\frac{1}{x_{2}}\left[x_{2}\left(r_{2}-k_{2} w^{s-1}\left(x_{2}\right)-b_{21} p\left(x_{1}\right)\right)-x_{2} h_{2} m^{t-1}\right] \\
& +x_{1} k_{1} \sum_{i=2}^{n}(i-1) a_{i} x_{1}^{i-1}+h_{1} \sum_{k=2}^{s}(k-1) b_{k} x_{1}^{k-1} \\
& +x_{2} k_{2} \sum_{k=2}^{q}(k-1) g_{k} x_{2}^{k-1}+h_{2} \sum_{j=2}^{z}(j-1) b_{j} k_{2}^{j-1} .
\end{aligned}
$$

Factorizando $-\operatorname{div}\left(f_{1}, f_{2}\right)$ se tiene que

$$
\begin{aligned}
-\operatorname{div}\left(f_{1}, f_{2}\right)= & -\frac{1}{x_{1}} f_{1}-\frac{1}{x_{2}} f_{2}+x_{1} k_{1} \sum_{i=2}^{n}(i-1) a_{i} x_{1}^{i-1}+h_{1} \sum_{k=2}^{s}(k-1) b_{k} x_{1}^{k-1} \\
& +x_{2} k_{2} \sum_{k=2}^{q}(k-1) g_{k} x_{2}^{k-1}+h_{2} \sum_{j=2}^{z}(j-1) b_{j} k_{2}^{j-1} .
\end{aligned}
$$

Entonces, la función $c\left(x_{1}, x_{2}\right)$ está dada por:

$$
\begin{aligned}
c\left(x_{1}, x_{2}\right)= & -x_{1} k_{1} \sum_{i=2}^{n}(i-1) a_{i} x_{1}^{i-1}-h_{1} \sum_{k=2}^{s}(k-1) b_{k} x_{1}^{k-1} \\
& -x_{2} k_{2} \sum_{k=2}^{q}(k-1) g_{k} x_{2}^{k-1}-h_{2} \sum_{j=2}^{z}(j-1) b_{j} k_{2}^{j-1} .
\end{aligned}
$$

De manera análoga al caso anterior se construye la siguiente ecuación diferencial parcial

$$
f_{1} \frac{\partial \Phi}{\partial x_{1}}+f_{2} \frac{\partial \Phi}{\partial x_{2}}=\Phi\left(c\left(x_{1}, x_{2}\right)-\operatorname{div}\left(f_{1}, f_{2}\right)\right) .
$$

Sea $\Phi=h(z)$, donde $z=z\left(x_{1}, x_{2}\right)$. Aplicando la regla de la cadena a (12) se tiene que:

$$
\left[f_{1} \frac{\partial z}{\partial x_{1}}+f_{2} \frac{\partial z}{\partial x_{2}}\right] \frac{\partial h}{\partial z}=h(c-\operatorname{div} F) .
$$

Así que,

$$
\frac{\frac{\partial h}{\partial z}}{h}=\frac{(c-\operatorname{div} F)}{\left[f_{1} \frac{\partial z}{\partial x_{1}}+f_{2} \frac{\partial z}{\partial x_{2}}\right]} .
$$




$$
\begin{gathered}
\frac{\partial \ln h}{\partial z}=\frac{-\frac{x_{2} f_{1}+x_{1} f_{2}}{x_{1} x_{2}}}{\left[f_{1} x_{2}+f_{2} x_{1}\right]}, \\
\frac{\partial \ln h}{\partial z}=-\frac{1}{z} .
\end{gathered}
$$

Por lo tanto,

$$
h\left(x_{1}, x_{2}\right)=\frac{1}{x_{1} x_{2}} .
$$

es una función de Dulac para el sistema (10).

Finalmente, se demostrará de manera análoga a los casos anteriores que el caso $c^{\prime}$ ) admite una función de Dulac.

Dado el sistema

$$
\begin{aligned}
& \dot{x_{1}}=x_{1} r_{1}\left[1-\frac{p_{1}\left(x_{1}\right)}{k_{1}+b_{12} p_{6}\left(x_{2}\right)}\right]-b p_{2}\left(x_{1}\right), \\
& \dot{x_{2}}=x_{2} r_{2}\left[1-\frac{p_{7}\left(x_{2}\right)}{k_{2}+b_{21} p_{3}\left(x_{1}\right)}\right]-c p_{8}\left(x_{2}\right),
\end{aligned}
$$

defínase

$$
f_{1}=x_{1} r_{1}\left[1-\frac{p_{1}\left(x_{1}\right)}{k_{1}+b_{12} p_{6}\left(x_{2}\right)}\right]-b p_{2}\left(x_{1}\right)
$$

$\mathrm{y}$

$$
f_{2}=x_{2} r_{2}\left[1-\frac{p_{7}\left(x_{2}\right)}{k_{2}+b_{21} p_{3}\left(x_{1}\right)}\right]-c p_{8}\left(x_{2}\right) .
$$

Calculando el negativo de la divergencia del sistema se tiene que

$$
\begin{aligned}
-\operatorname{div}\left(f_{1}, f_{2}\right)= & -r_{1}+\frac{p_{1}\left(x_{1}\right) r_{1}}{k_{1}+b_{12} p_{6}\left(x_{2}\right)}+\frac{x_{1} p_{1}^{\prime}\left(x_{1}\right) r_{1}}{k_{1}+b_{12} p_{6}\left(x_{2}\right)}+b p_{2}^{\prime}\left(x_{1}\right)-r_{2} \\
& +\frac{p_{7}\left(x_{2}\right) r_{2}}{k_{2}+b_{21} p_{3}\left(x_{1}\right)}+\frac{x_{2} p_{7}^{\prime}\left(x_{2}\right) r_{2}}{k_{2}+b_{21} p_{3}\left(x_{1}\right)}+c p_{8}^{\prime}\left(x_{2}\right) .
\end{aligned}
$$

Derivando los polinomios se tiene que

$$
\begin{aligned}
& p_{2}^{\prime}\left(x_{1}\right)=\left(b_{1}+b_{2} x_{1}+b_{3} x_{1}^{2}+\cdots+(s-1) b_{s} x_{1}^{s-1}\right)+\sum_{k=2}^{s}(k-1) b_{k} x_{1}^{k-1} \\
& p_{8}^{\prime}\left(x_{2}\right)=\left(k_{1}+k_{2} x_{2}+k_{3} x_{2}^{2}+\cdots+(r-1) k_{r} x_{2}^{r-1}\right)+\sum_{j=2}^{r}(j-1) k_{j} x_{2}^{j-1} .
\end{aligned}
$$


Considérense los siguientes cambios de variables:

$$
\begin{aligned}
\left(b_{1}+b_{2} x_{1}+b_{3} x_{1}^{2}+\cdots+(s-1) b_{s} x_{1}^{s-1}\right) & =w^{t-1}\left(x_{1}\right), \\
\left(k_{1}+k_{2} x_{2}+k_{3} x_{2}^{2}+\cdots+(j-1) k_{j} x_{2}^{j-1}\right) & =h^{j-1}\left(x_{2}\right) .
\end{aligned}
$$

Así se tiene que:

$$
\begin{aligned}
& p_{2}^{\prime}\left(x_{1}\right)=w^{t-1}\left(x_{1}\right)+\sum_{k=2}^{s}(k-1) b_{k} x_{1}^{k-1} \\
& p_{8}^{\prime}\left(x_{2}\right)=h^{j-1}\left(x_{2}\right)+\sum_{j=2}^{r}(j-1) k_{j} x_{2}^{j-1}
\end{aligned}
$$

Con los cambios de variables propuestos se tiene que la divergencia del campo vectorial está dada por:

$$
\begin{aligned}
-\operatorname{div}\left(f_{1}, f_{2}\right)= & -\frac{1}{x_{1}}\left[r_{1} x_{1}\left(1-\frac{p_{1}\left(x_{1}\right)}{k_{1}+b_{12} p_{6}\left(x_{2}\right)}\right)-b x_{1} w^{t-1}\left(x_{1}\right)\right] \\
& +\frac{p_{1}^{\prime}\left(x_{1}\right) r_{1}}{k_{1}+b_{12} p_{1}\left(x_{2}\right)}+b \sum_{k=2}^{s}(k-1) b_{k} x_{2}^{k-1} \\
& -\frac{1}{x_{2}}\left[r_{2} x_{2}\left(1-\frac{p_{2}\left(x_{2}\right)}{k_{2}+b_{21} p_{3}\left(x_{1}\right)}\right)-c x_{2} h^{j-1}\left(x_{2}\right)\right] \\
& +\frac{p_{2}^{\prime}\left(x_{2}\right) r_{2}}{k_{2}+b_{21} p_{3}\left(x_{1}\right)}+c \sum_{j=2}^{r}(j-1) k_{j} x_{2}^{j-1} .
\end{aligned}
$$

Después de factorizar $-\operatorname{div}\left(f_{1}, f_{2}\right)$ se tiene que

$$
\begin{aligned}
-\operatorname{div}\left(f_{1}, f_{2}\right)= & -\frac{1}{x_{1}} f_{1}-\frac{1}{x_{2}} f_{2}+\frac{p_{1}^{\prime}\left(x_{1}\right) r_{1}}{k_{1}+b_{12} p_{1}\left(x_{2}\right)}+b \sum_{k=2}^{s}(k-1) b_{k} x_{2}^{k-1} \\
& +\frac{p_{2}^{\prime}\left(x_{2}\right) r_{2}}{k_{2}+b_{21} p_{3}\left(x_{1}\right)}+c \sum_{j=2}^{r}(j-1) k_{j} x_{2}^{j-1} .
\end{aligned}
$$

Si la función $c\left(x_{1}, x_{2}\right)$ está dada por

$$
\begin{aligned}
c\left(x_{1}, x_{2}\right)= & -\frac{p_{1}^{\prime}\left(x_{1}\right) r_{1}}{k_{1}+b_{12} p_{1}\left(x_{2}\right)}-b \sum_{k=2}^{s}(k-1) b_{k} x_{2}^{k-1} \\
& -\frac{p_{2}^{\prime}\left(x_{2}\right) r_{2}}{k_{2}+b_{21} p_{3}\left(x_{1}\right)}-c \sum_{j=2}^{r}(j-1) k_{j} x_{2}^{j-1} .
\end{aligned}
$$


Entonces la ecuación diferencial parcial (12) se reduce a

$$
\frac{\partial \ln h}{\partial z}=-\frac{1}{z}
$$

cuya solución,

$$
h\left(x_{1}, x_{2}\right)=\frac{1}{x_{1} x_{2}},
$$

es una función de Dulac para el sistema (13), por lo que se demuestra el resultado.

\section{Ejemplos}

En esta sección se muestra la aplicación de los resultados presentados en este trabajo a modelos de la biomatemática.

En el ejemplo para fitoplancton y zooplancton mostrado en (8) si el efecto negativo sobre el zooplancton debido a la presencia de toxinas liberadas por el fitoplancton es descrito por una respuesta de Holling de tipo III como la usada en [11] se tiene el siguiente modelo

$$
\begin{aligned}
& \dot{p}=r p\left[1-\frac{p}{k}\right]-\alpha z p \\
& \dot{z}=\beta p z-c z-\frac{\theta p^{2}}{\gamma+p^{2}} z
\end{aligned}
$$

Nótese que el modelo (14), es una generalización del ejemplo 1. Así que el modelo admite una función de Dulac, por lo que no existirán soluciones periódicas del modelo.

Si en el ejemplo de competencia y captura dado por (10) se usa un término cuadrático para describir la captura de la especies en lugar de un término lineal, es decir, cuando la población es pequeña se capturan muy pocos peces y cuando la población es grande se capturan mucho más peces, se tiene el siguiente modelo

$$
\begin{aligned}
& \dot{x_{1}}=x_{1}\left(r_{1}-k_{1} p_{1} x_{1}\right)-b_{1} x_{1} x_{2}-h_{1} x_{1}^{2}, \\
& \dot{x_{2}}=x_{2}\left(r_{2}-k_{2} p_{2} x_{2}\right)-b_{2} x_{1} x_{2}-h_{2} x_{2}^{2} .
\end{aligned}
$$


Usando el resultado $b^{\prime}$ ) del teorema 1 se concluye que el sistema (15) no tiene soluciones periódicas debido que el sistema admite una función de Dulac.

Finalmente, si en el modelo de mutualismo dado por (3) se supone una captura proporcional a la población, se tiene el siguiente modelo

$$
\begin{aligned}
& \dot{x_{1}}=r_{1} x_{1}\left[1-\frac{x_{1}}{k_{1}+b_{1} x_{2}}\right]-h_{1} x_{1}, \\
& \dot{x_{2}}=r_{2} x_{2}\left[1-\frac{x_{2}}{k_{2}+b_{2} x_{1}}\right]-h_{2} x_{2},
\end{aligned}
$$

el cual admite una función de Dulac al ser un caso particular de c'). Así que el modelo no presenta soluciones periódicas.

\section{Discusión}

La existencia de órbitas periódicas asociadas a modelos de interacciones entre especies es un problema que permanece abierto y que ha sido abordado en numerosas ocasiones. Conocer si la dinámica de las poblaciones está descrita por oscilaciones sostenidas o no es de vital interés para la ecología. Lo anterior es debido a que la periodicidad en muchos casos está presente en los ciclos vitales de muchos organismos. Un ejemplo donde la periodicidad beneficia a un organismo es dado por el tántalo americano o cigüeña de cabeza pelada ( $M y c$ teria americana), la cual se reproduce al inicio de la temporada seca (invierno). En ese sentido, la cigüeña de bosque es beneficiada por la periodicidad de las estaciones del año ya que los ecosistemas de nivel fluctuante de agua donde la cigüeña busca alimento se ven afectados al inicio del invierno debido a que los niveles de agua comienzan a descender y los peces de los que se alimentan están concentrados en pozas aisladas lo que favorece la captura de alimento. De no ser este el caso, es decir, si en la temporada seca los niveles de agua no fueran bajos las cigüeñas no llegarían a anidar lo cual sería catastrófico para su población [3]. Más aún, la periodicidad puede ser vista como una estrategia que las poblaciones pueden adoptar para favorecer su crecimiento, por ejemplo, las cigarras periódicas (Magicicada), las cuales pasan aproximadamente 13 o 17 años de su vida bajo la tierra en la clase juvenil y después entre 2 y 4 semanas de su vida emergen de la tierra ya como adultos, buscan reproducirse en estas últimas semanas [12], es decir, se reproducen en períodos de 13 o 14 años. Este mecanismo de reproducción permite que los periodos de reproducción de las cigarras y la mayoría de sus depredadores (aves, hormigas, arañas, reptiles y algunos mamíferos pequeños) no estén sincronizados ya que la mayoría de sus depredadores tiene un ciclo de vida de entre 2 y 3 años. En este ejemplo, el tiempo que dura el ciclo de 
vida de la cigarra es de vital importancia para el aseguramiento de su población, la cual no logra estar a salvo de todos los peligros ya que aun cuando el ciclo de vida de las cigarras es muy largo, estas pueden ser infectadas por un parásito fúngico especializado el cual secuestra las señales sexuales de cigarras periódicas, lo que permite que dicho hongo encuentre nuevos hospederos a expensas de las cigarras, los cuales no se reproducen con éxito [2].

Por otro lado, no siempre la existencia de periodicidad en los ciclos poblacionales es benéfica para la población ya que si existen oscilaciones periódicas y la amplitud de dichas oscilaciones es suficientemente grande las poblaciones pueden estar por debajo de umbrales críticos de población, lo que es catastrófico para las poblaciones. Caso contrario a cuando no existen oscilaciones sostenidas en las poblaciones y estas tienden a sus respectivos equilibrios.

Si bien, los modelos clásicos de la ecología matemática han sido ampliamente estudiados, y se conoce su dinámica en totalidad, conocer si perturbaciones del sistema generan órbitas periódicas es un problema vigente. En este trabajo se demuestra que algunas generalizaciones de modelos usados comúnmente excluyen la existencia de órbitas periódicas. Dichas generalizaciones pueden describir mecanismos de interacción entre las especies o mecanismos que describan manejo de ellas. Además, dichos resultados pueden ser usados para demostrar estabilidad de los puntos de equilibrios al ser usados junto con el teorema de Poincaré-Bendixon. Ambas aplicaciones son fundamentales para describir la dinámica de un modelo poblacional. En trabajo futuro se buscará construir funciones de Dulac para modelos describiendo otro tipo de interacciones entre especies.

\section{Agradecimientos}

Los autores agradecen las apreciables observaciones de los referees anónimos que ayudaron a mejorar el presente trabajo.

\section{Financiamiento}

Mendoza Emmanuel agradece al CONACYT por la beca de posgrado asignada. Osvaldo Osuna agradece el soporte económico por parte de la CIC de la Universidad Michoacana. Villavicencio-Pulido Geiser agradece el soporte económico por parte de la Departamento de Ciencias Ambientales de la Universidad Autónoma Metropolitana. 


\section{Referencias}

[1] L. Cherkas, A. Grin A., Algebraic aspects of finding a Dulac function for polynomial autonomous systems on the plane, Differ. Equa., 37(2001), no. 3, 411-417, Doi: 10.1023/A:1019250717290

[2] J.R. Cooley, D.C. Marshall, K.B.R. Hill, A specialized fungal parasite (Massospora cicadina) hijacks the sexual signals of periodical cicadas (Hemiptera: Cicadidae: Magicicada), Scientific Reports 8(2018), 1432, Doi: $10.1038 /$ s41598-018-19813-0

[3] M.P. Kahl Jr., Food ecology of the Wood Stork (Mycteria Americana) in Florida, Ecological Monographs 34(1964), no. 2, 97-117. Doi: 10. $2307 / 1948449$

[4] J.D. Murray, Mathematical Biology - I. An Introduction, Springer-Verlag, New York, 2002. Doi: 10.1007/b98868

[5] O. Osuna, C. Vargas, Construction of Dulac functions for mathematical models in population biology, International Journal of Biomathematics, 8(2015), no. 03. Doi: 10.1142 /S1793524515500357

[6] O. Osuna, G. Villaseñor, On the Dulac functions, Qualitative Theory of Dynamical Systems 10(2011), no. 1, 43-49. Doi: 10.1007/ s12346-011-0036-y

[7] O. Osuna, G. Villaseñor, Some properties of the Dulac functions set, E.J. of Qualitative Theory of Differential Equations 72(2011), 1-8. Doi: 10 . 14232 /ejqt de.2011.1.72

[8] O. Osuna, G. Villavicencio, Generating Dulac functions for differential equations and biological models, Avanza 6(2016) FM-IIT, UACJ, 1-8.

[9] E. Saez, I. Szántó, On the construction of certain Dulac function, Trans. Automat. Control 33(1988), no. 9, 856. Doi: $10.1109 / 9.1315$

[10] R. Sarkar, J. Chattopadhayay, Occurrence of planktonic blooms under environmental fluctuations and its possible control mechanismMathematical models and experimental observations, Journal of Theoretical Biology, 224(2003), no. 4, 501-516. Doi: 10.1016/ S0022-5193(03)00200-5 
[11] M. Scheffer, Fish and nutrients interplay determines algal biomass: A minimal model, Oikos 62(1991), no. 3, 271-282. Doi: $10.2307 / 3545491$

[12] T. Sota, S. Yamamoto, J.R. Cooley, K.B.R. Hill, C. Simon, J. Yoshimura, Independent divergence of 13- and 17-y life cycles among three periodical cicada lineages, PNAS 110(2013), no. 17, 6919-6924. Doi: $10.1073 /$ pnas. 1220060110

[13] E. Venturino, The effects of the diseases on competing species, Mathematical Biosciences 174(2001), no. 2, 111-131. Doi: 10.1016/ S0025-5564(01)00081-5

\section{A Apéndice}

Sea $\Omega \subset \mathbb{R}^{2}$ una región simplemente conexa y

$$
\begin{aligned}
& \dot{x_{1}}=f_{1}\left(x_{1}, x_{2}\right), \\
& \dot{x_{2}}=f_{2}\left(x_{1}, x_{2}\right) .
\end{aligned}
$$

Considérese el conjunto de la funciones continuas $C^{0}(\Omega, \mathbb{R})$ y defínanse los siguientes conjuntos:

$\mathcal{F}_{\Omega}=\left\{f \in C^{0}\left(\Omega, \mathbb{R}^{ \pm} \cup\{0\}\right)\right.$ : se anula a lo más en un conjunto de medida cero $\}$,

$$
\mathcal{D}_{\Omega}^{+}=\left\{\Phi \in C^{1}(\Omega, \mathbb{R}): k=\frac{\partial\left(\Phi f_{1}\right)}{\partial x_{1}}+\frac{\partial\left(\Phi f_{2}\right)}{\partial x_{2}} \geq 0, k \in \mathcal{F}_{\Omega}\right\}
$$

$\mathrm{y}$

$$
\mathcal{D}_{\Omega}^{-}=\left\{\Phi \in C^{1}(\Omega, \mathbb{R}): k=\frac{\partial\left(\Phi f_{1}\right)}{\partial x_{1}}+\frac{\partial\left(\Phi f_{2}\right)}{\partial x_{2}} \leq 0, k \in \mathcal{F}_{\Omega}\right\} .
$$

Una función de Dulac en el sistema (17) es un elemento en el conjunto:

$$
\mathcal{D}_{\Omega}(F):=\mathcal{D}_{\Omega}^{+}(F) \cup \mathcal{D}_{\Omega}^{-}(F) .
$$

Osuna y Vargas en [5] establecen el siguiente resultado.

Teorema 2 Si existe un función $c \in \mathcal{F}_{\Omega}$ tal que $\Phi$ es una solución del sistema

$$
f_{1} \frac{\partial \Phi}{\partial x_{1}}+f_{2} \frac{\partial \Phi}{\partial x_{2}}=\Phi\left(c\left(x_{1}, x_{2}\right)-\operatorname{div}\left(f_{1}, f_{2}\right)\right),
$$

con $\Phi \in \mathcal{F}_{\Omega}$, entonces $\Phi$ es una función de Dulac para (17) sobre $\Omega$. 